\title{
Research on the Strategy of Promoting High-quality Development of Agriculture in Henan Province
}

\author{
Xitao Dong \\ Department of Marketing, School of Economics and Management, Pingdingshan University, \\ Pingdingshan 467000, China \\ 00094203@163.com
}

\begin{abstract}
China's economy has changed from the stage of high-speed growth to the stage of high-quality development. As an important part of the economy, the development strategy must change accordingly. Henan Province is an important agricultural province in China, and its agricultural economic development plays an important role in the country. Henan Province has good climatic conditions, natural environmental resources and labor resources, and has made good achievements in agricultural development. However, there are still some problems, such as low degree of agricultural industrialization, deterioration of agricultural ecological environment, slow progress of agricultural technology, hidden problems in the quality and safety of agricultural products, and slow growth of farmers' income, Henan Province needs to effectively deal with it by cultivating and developing agricultural leading industries and new types of agricultural businesses, and promoting the progress of agricultural technology.
\end{abstract}

Keywords: High-quality development of agriculture, Henan Province, Agricultural development strategy.

\section{Introduction}

In 2017, the 19th National Congress of the Communist Party of China put forward the new expression of "high-quality development" for the first time, indicating that China's economy has shifted from the stage of high-speed growth to the stage of high-quality development. Since the expression of "high-quality development" was first put forward, the domestic research on high-quality development in economy, region, industry, society and other aspects has shown explosive growth. At present, the research related to agricultural high-quality development mainly includes the connotation and evaluation of agricultural high-quality development.

Henan Province is an important agricultural province in China. It is an important national commodity grain base and the most important grain production area and animal husbandry base. The development of agricultural economy in Henan Province plays an important role in the country. Therefore, studying high-quality development of agriculture in Henan Province, finding effective countermeasures to promote high-quality development in Henan Province, which is very important for promoting provincial agriculture sustainable development and improving agricultural competitiveness.

\section{The Current Situation of Agricultural Development in Henan Province}

The cultivated land area of Henan Province is 8158.29 thousand hectares, ranking second in China. The total sown area is 146764300 hectares, mainly grain crops. The grain planting area is 107345400 hectares. The grain output of Henan Province is 66.9536 million tons, accounting for nearly one tenth of China's total grain output. It is one of the three provinces with a national grain output of more than 30 million tons. The output of peanut and sesame ranks first in China, the output of cotton is 27100 tons and the output of oil-bearing crops is 6.4545 million tons. The output of vegetables and edible fungi is 72.0667 million tons, and the output of fruit is 15.8537 million tons. Henan Province has a large wheat planting area, accounting for $54 \%$ of the grain sowing area, and its output accounts for more than a quarter of the country, ranking first in the country. Corn and tuber crops are the second and third largest grain crops in Henan Province. Rice is the fourth largest grain crop in Henan Province, planting area accounts for $4 \%$ of the province's food area, and its output accounts for $8 \%$ of total production. Henan is one of the main soybean producing areas in China and the second largest soybean producing province in China. Henan is one of the main cotton producing areas in China, and the total cotton output ranks second in China. The total output of tobacco leaf has always ranked first in China, and it is the largest tobacco leaf production base in China. The production of oil-bearing crops in Henan mainly includes peanut, rapeseed and sesame, and its sowing area rank third among provinces and regions in China.

\subsection{Resource Endowment of Agriculture in Henan Province}

\subsubsection{Geographical Overview}

Henan is located between $31^{\circ} 23^{\prime}-36^{\circ} 22^{\prime} \mathrm{N}$ and $110^{\circ} 21^{\prime}-116^{\circ}$ $39^{\prime} \mathrm{E}$. The total area of the province is 167000 square kilometers, accounting for $1.73 \%$ of the total area of the country. The terrain is high in the West and low in the East. Taihang Mountain, Funiu Mountain, Tongbai mountain and Dabie Mountain are distributed in a semi ring along the provincial boundary in the north, West and south. The Middle East is the Alluvial Region of Huang-Huai-Hai Plain and the southwest is the Nanyang Basin. Plain \& basins, mountains \& hills account for $55.7 \%$ and $44.3 \%$ of the total area respectively. Henan Province has diverse terrain, convenient transportation and fertile land. 


\subsubsection{Weather and climate}

Most areas of Henan Province are located in the warm temperate zone, and the southern area is located in the subtropical zone. It belongs to the continental monsoon climate from the north subtropical zone to the warm temperate zone. At the same time, it also has the characteristics of the transition from plain to hilly and mountainous climate. In recent 10 years, the annual average temperature in the province is $12.9-16.5^{\circ} \mathrm{C}$, the annual average precipitation is $464.2-1193.2 \mathrm{~mm}$, the annual average sunshine hours are 1505.9-2230.7 hours, and the annual average frost-free period is 208.7-290.2 days, which is suitable for the growth of a variety of crops.

\subsubsection{Cultivated land and water resources}

The cultivated land area of the province is 122.29 million mu, with a per capita cultivated land of $1.12 \mathrm{mu}$. Henan is the only province in China that crosses the Yangtze River, Huaihe River, Yellow River and Haihe River. Most of the rivers in the province originate from the mountains in the west, northwest and Southeast. 560 rivers have a drainage area of more than 100 square kilometers; 64 rivers have a drainage area of 1000 square kilometers and above; 11 rivers have a drainage area of 10000 square kilometers and above. The total amount of water resources in the province is 40.353 billion cubic meters, and the per capita water resources is about 368 cubic meters, less than $1 / 5$ of the national average level. It is a province with serious water shortage. Water resources have formed a certain degree of constraints on agricultural development.

\subsection{Labor Resources}

According to the results of the seventh national census, among the permanent residents in Henan Province, 44286965 live in rural areas, accounting for $44.57 \%$. Compared with the sixth national census in 2010, the urban population increased by 18858787 , the rural population decreased by 13523207 , and the proportion of urban population increased by 16.91 percentage points. The number of labor force in Henan Province is huge, but the rural population has decreased significantly. Most of the workers who make a living in agriculture in rural areas are the elderly. Young and middle-aged farmers work in cities, and farmers' concurrent business behavior is very common. Generally speaking, the overall quality of farmers is not high. It is mainly reflected in the following aspects: farmers have less years of education, lack of scientific and technological knowledge, weak awareness of market competition and low labor productivity. In addition, the level of farmers' labor skills is also low, and the training in agricultural technology is insufficient. Many farmers do not have any modern agricultural professional skills, and mainly rely on traditional production methods to engage in agricultural production. The low quality of labor force has seriously restricted the economic growth and development of agriculture in Henan Province. At the same time, with the continuous development of new types of agricultural businesses, the agricultural production in Henan Province shows the trend of large-scale, specialization and industrialization, which also invests new vitality into the upgrading of agricultural industry in Henan Province.

\subsection{Agricultural Production Equipment}

By the end of 2019, the total power of agricultural machinery in Henan Province was 103.5697 million KW, an increase of $1.49 \%$ over the previous year; The number of large and medium-sized agricultural tractors and supporting agricultural tools for large and medium-sized tractors reached 373074 and 654206 respectively, an increase of $7.27 \%$ and $3.54 \%$ respectively; The number of primary processing machines for agricultural products reached 571200 , and the annual rural power consumption was 35.383 billion $\mathrm{kwh}$, an increase of $0.26 \%$. The electrification and mechanization of agricultural production and agricultural product processing have been basically realized.

\section{The Problems Existing in the High-quality Development of Agriculture in Henan Province}

\subsection{Low Degree of Agricultural Industrialization}

At present, most of the agricultural production in Henan Province is still in a decentralized small-scale peasant economy. The production scale is small, the degree of organization is low, the scale of leading enterprises is small, the radiation driving ability is weak, and the processing and conversion rate of agricultural products is not high. The development of new types of agricultural businesses is slow, their industrial integration ability is not strong, and their ability to resist market risks is insufficient.

\subsection{Deterioration of Agricultural Ecological Environment}

For a long time, due to industrial pollution and insufficient attention to environmental protection, a large area of farmland topography has changed, the atmospheric environment, soil environment, surface and groundwater quality have been polluted, coupled with the unreasonable use of agricultural inputs such as chemical fertilizers and pesticides, the soil structure has changed, the nutrient composition is unbalanced, and harmful substances have accumulated. These ecological environment changes have brought serious impact on the sustainable development of agriculture.

\subsection{The Level of Agricultural Production Technology is Relatively Low}

The agricultural technology extension system is not perfect, the agricultural technology extension force is weak, and the development of modern agricultural science and technology parks lags behind. Agricultural science and technology park is a window to show modern agriculture, a demonstration education base for modern agricultural science and technology and business philosophy, a link between scientific research and agricultural production, and a pioneer in the construction of urban agriculture. But the number of agricultural science and technology demonstration parks in Henan Province is still relatively small, and its demonstration and educational role has not been brought into full play.

\subsection{The Quality of Agricultural Products Needs to be Improved}


The quality inspection system of agricultural products is not perfect. Due to the large number of small farmers, it is impossible to comprehensively test their agricultural products. The construction of quality inspection center also needs to be improved, which can not meet the needs of comprehensive supervision of agricultural products. The agricultural standardized production capacity is not high, the irrational use of pesticides and chemical fertilizers is common, and the safety level and market competitiveness of agricultural products need to be further improved.

\subsection{Farmers' Income is not Growing Fast}

In 2021, the per capita disposable income of rural residents in Henan Province was 16107.9 yuan, the national average was 17131.5 yuan, ranking 19th among 31 provinces and cities in China (excluding Hong Kong, Macao and Taiwan), fifth among the six provinces in Central China, only higher than that of Jiangxi Province. Due to the large-scale rural population in Henan Province, the per capita disposable income will be low, but it also shows that the added value of agricultural production in Henan Province is not high.

\section{The Suggestions on Accelerating the High-quality Development of Agriculture in Henan Province}

\subsection{Cultivate and Develop Agricultural Leading Industries}

On the basis of ensuring national food security, we will strive to optimize the industrial structure and product structure. Henan should firmly establish the market concept, implement the strategy of "storing grain in the land and technology", and coordinate the relationship between output and production capacity, increase production and increase income. On the basis of stabilizing the planting area of grain crops, steadily improve the comprehensive agricultural production capacity, promote the production of high-quality strong gluten wheat in Northern Henan and the Middle East of Henan, and promote the production of high-quality weak gluten wheat along the Huaihe River in southern Henan; Appropriately reduce the corn planting area; Promote the construction of high-quality rice production bases in areas rich in water resources such as Xinyang and Xinxiang; Increase the coverage of improved peanut varieties along the Yellow River; Adjust the planting area of peanut with high oil and high oleic acid; Accelerate the construction of facility vegetable, promote industrial seed culture, and develop advantageous new varieties; Accelerate the construction of high-quality agricultural product bases such as fruits, tea, edible fungi, traditional Chinese medicine and aquatic products, and comprehensively promote the regionalization of layout and production standardization.

\subsection{Promote the Development of Agricultural Leading Enterprises}

Grain, fruits, vegetables, meat products, dairy products and other processing industries should have breakthrough development, and the proportion of the output value of agricultural and sideline products processing enterprises in the total industrial output value continues to rise. In accordance with the principle of "supporting the excellent, the strong and the big", cultivate and expand leading enterprises, accelerate capital agglomeration, promote the integration of resources and brands, develop a number of agricultural processing enterprise clusters, and form a number of backbone agricultural enterprises with an annual sales revenue of more than 50 million yuan.

\subsection{Develop Agricultural Products Logistics Industry}

Focus on building a number of large-scale wholesale trading markets for agricultural products, and form a modern logistics distribution center and distribution center for agricultural products with wide radiation and large throughput. Strengthen the transformation and upgrading of the existing agricultural product market and Logistics Industrial Park, and introduce new forms such as e-commerce, futures and auction. We will vigorously cultivate large agricultural product marketers and brokers, cultivate rural circulation enterprises, and broaden the circulation channels of agricultural products.

\subsection{Develop New Agricultural Business Entities}

We should actively encourage and support the development of rural cooperative economic organizations, adhere to the principle of "farmers' voluntariness, democratic management, risk sharing and benefit sharing", and improve farmers' organization degree and self-management and self-service ability through various forms such as technology alliance, production alliance, circulation alliance and processing alliance, so as to make them truly become the main body of the market, and Obtain rich postpartum value-added profits in the process of extending the industrial chain. By establishing and improving the circulation mechanism of management right and use right of agricultural land, we can change the pattern of small-scale and decentralized agricultural production and form an appropriate scale of management.

\subsection{Promote Agricultural Science and Technology Progress and Application}

The quality of agricultural workers is the most important guarantee for the high-quality development of agriculture. We should take technical training for farmers and improve the quality of agricultural workers as a long-term important work. Comprehensively promoting the progress of agricultural science and technology needs to improve the quality of workers and the demonstration and popularization of modern agricultural science and technology.

First, we should comprehensively carry out a series of activities, such as new farmers' science and technology training, green certificate training, labor transfer training, etc. Through various training courses, on-site lectures and on-site demonstrations by experts and scientific and technological personnel, all kinds of rural talents are trained, so that every farmer has a skill. Second, strengthen ties with colleges and universities and scientific research institutes inside and outside the province. Through the introduction of talents, projects and joint tackling of key problems, we will strengthen cooperative research in agricultural information technology, biotechnology and other scientific and technological fields. Speed up the application of high technology in agriculture. 
Third, accelerating the construction of modern agricultural demonstration parks. Focus on supporting a number of high-tech agricultural parks with high technology content, good benefits and strong radiation driving ability. These agricultural parks have become the forerunners of promoting the progress of agricultural science and technology, promoting the transformation of agricultural management ideas, improving the agricultural development environment, and accelerating the transformation from traditional agriculture to modern agriculture. Fourth, strengthening the popularization of agricultural technology, such as establishing a new agricultural technology extension system, vigorously promoting advanced and practical agricultural technology, accelerating the transformation of agricultural scientific and technological achievements and promoting the overall improvement of agricultural technology popularization.

\subsection{Strengthen Agricultural Exchanges and Cooperation with Developed Regions}

In order to make Henan agriculture in line with international standards, we must strengthen agricultural opening to the outside world. Henan should actively introduce foreign high and new agricultural technologies, such as standardized production, preservation, processing, quality inspection of agricultural products, animal and plant epidemic prevention and quarantine, food safety and health technology, etc. Promote the development of foreign exchange earning agriculture through the agricultural technology introduction demonstration area and international agricultural exchange experimental area in Henan. These demonstration and experimental zones can strengthen cooperation and exchanges in the production, processing and circulation of new agricultural varieties, new equipment and agricultural products. So as to improve the competitiveness of agricultural products in Henan Province in entering the international market.

\subsection{Increase Investment in Agriculture}

Create a good investment environment and development conditions, adhere to the principle of "investors have ownership and operators obtain income", encourage overseas capital and private capital to participate in agricultural construction in various forms such as cooperation, equity participation, and sole proprietorship, especially pay attention to attracting industrial and commercial capital to invest in modern agriculture and form a diversified and multi-channel investment and financing mechanism. Continue to increase government investment and give full play to the guiding role of financial investment. Make some overall plans for agricultural special funds such as comprehensive agricultural development, "vegetable basket" project, agricultural infrastructure and agricultural national debt, and focus on increasing investment in agricultural scientific research, infrastructure, training of agricultural technicians and farmers, marketing of agricultural products, construction of information system and ecological environment. Continue to improve the agricultural security mechanism and establish an insurance security system with government support, mainly handled by cooperative organizations and wide participation of agricultural enterprises and farmers, so as to effectively reduce the natural and market risks of agriculture. Give full play to the positive role of various financial institutions, study and formulate credit policies to support agriculture, increase the supply of agricultural credit, and guide financial institutions to support high-quality development of agriculture.

\subsection{Strengthen the Construction of Agricultural Infrastructure}

In accordance with the principle of "comprehensive planning, overall consideration and addressing both symptoms and root causes", actively promote the construction of agricultural infrastructure supporting the high-quality development of agriculture. Combined with the comprehensive agricultural development projects in recent years, relying on the existing infrastructure conditions, carry out the construction of supporting facilities such as agricultural facilities, road network and environmental transformation, and speed up the development process of agricultural mechanization. Accelerate the construction of drinking water and water-saving irrigation projects for the masses in mountainous areas, vigorously develop rural household biogas and other biogas projects, and form infrastructure conditions and equipment advantages that meet the requirements of high-quality development agriculture.

\subsection{Promote the Construction of Agricultural Production Standardization}

Speed up the construction of agricultural production standard system, strengthen the implementation of agricultural standards, plan and manage agriculture with modern industrial ideas, and transform traditional agriculture with modern science and technology; Promote the change of agricultural production and operation scale, standardize the production and operation of agricultural products, and improve the agricultural organization mode with modern production organization mode; Optimize the regional distribution of advantageous agricultural products, and promote large-scale planting and breeding, standardized production, industrialized operation and scientific management.

\section{Acknowledgement}

This paper is the phased achievement of Henan Philosophy and Social Science Planning Project, Project No.: 2020BJJ052.

\section{References}

[1] Lu Yan, Tian Yingfang, Xu Bao, Li Hao, Li Xin. (2021). Study on the implementation path and guarantee mechanism of high quality development in Henan under the perspective of food security. Agricultural Science and Technology Management, 40 (01), 12-15.

[2] Zhang inventions, Dingfeng, Wang Ping. (2021). Evaluation and temporation of agricultural high quality development in China's property area. Zhejiang Agricultural Journal, 33 (01), 150-160.

[3] Lu Yuan, He Xiuqin, Lu He Ming. (2020). Strive to promote high quality development in Henan. Rural. Agriculture. Farmers (B Edition), (11), 33-34. 
[4] Jia Qiangfa. (2020). Analysis of high quality development in Henan. Henan Agriculture, (29), 57-58.

[5] Zhang Zhancang, Yang Shu Ting, Wang Jianguo, He Jianyi Committee, Chai Zhongchang, Luo Jianzhong. (2020). Study on the existing problems and strategic countermeasures in Henan Province. Innovation Technology, 20 (01), 30-36.

[6] Wang Xifeng, Shen Dajun. (2019). Influence of high quality development in the Yellow River Basin on Water Resource Carrying Capacity. Environmental Economic Research, 4 (04), 47-62.

[7] Xinling, An Xiaoning. (2019). Analysis of the construction and measurement of evaluation system of agricultural high quality development in my country. Economics, (05), 109-118. 